\title{
Optimal Design of the Sealing Structure of a Hydraulic Cylinder on the Basis of a Surrogate Model
}

\author{
Hong Zhang, Yu Sun (D), Chenchen Li, and Haoran Wang \\ School of Mechanical Engineering, Dalian University of Technology, Dalian 116024, China \\ Correspondence should be addressed to Yu Sun; 284274494@qq.com
}

Received 20 April 2020; Revised 22 June 2020; Accepted 24 July 2020; Published 26 August 2020

Academic Editor: Gloria Terenzi

Copyright ( 92020 Hong Zhang et al. This is an open access article distributed under the Creative Commons Attribution License, which permits unrestricted use, distribution, and reproduction in any medium, provided the original work is properly cited.

In this study, a finite-element simulation model was established for a two-dimensional hydraulic cylinder seal structure with highly nonlinear materials and contacts for achieving the optimal sealing effect with the structure. The effects of gaps as well as single and double O-rings on the sealing effect were examined. On the basis of this examination, a parametric model was developed for double-O-rings without gaps, which are suitable for hydraulic cylinder sealing. Suitable design variables, objective functions, and constraint conditions were determined for the parametric model. Then, a surrogate model was fitted and optimised through a constrained Latin hypercube method, an interpolating recmultiquadric radial basis function method, and the genetic algorithm. The results indicate that the seal and seal groove structures obtained through optimisation with the surrogate model provide a superior sealing effect to unoptimised structures. Therefore, the combination of the developed surrogate model and finite-element method can provide a theoretical reference for the design of the sealing structure of hydraulic cylinders.

\section{Introduction}

Hydraulic transmission has attracted considerable attention for transportation vehicles, such as automobiles, ships, and aircraft, due to the development of manufacturing with the advancement of technology. In a hydraulic system, structures such as valves, pumps, and cylinders are connected to hydraulic pipe joints through a piping system. Hydraulic pipe joints, pumps, valves, and cylinders are the most basic hydraulic system components that play a vital role in the circulation of gas, water, and oil in the system [1-5]. Because the hydraulic pipeline is in a high-risk environment in the circulation and power system, the pipeline seal requires further analysis [6]. In aircraft, ships, automobiles, and other transportation vehicles, when the hydraulic seal system fails, internal oil, gas, pollutants, and waste are leaked. Such failure may also cause fatalities and major accidents. Thus, the sealing performance of the hydraulic pipeline sealing structure in vehicles considerably influences the safety and reliability of the equipment $[7,8]$.

Currently, the research on and production capacity of seals in China is relatively low, and manufacturers blindly increase the compression amount of seals in most cases to solve the problem of hydraulic seal failure [9]. Although the sealing purpose is temporarily achieved when using the aforementioned approach, the seal duration and reliability decrease, which may cause problems such as seal damage. Therefore, research on the sealing performance of hydraulic cylinder sealing structures is critical [10].

In general, the functional components of a sealing device are the upper and lower flanges and the middle sealing rubber. Therefore, a study of the sealing performance should begin from the analysis of these three components. The amount of compression of the sealing rubber directly affects the sealing performance of the hydraulic cylinder sealing structure. Moreover, large compression reduces the service life of the sealing rubber. The cross-sectional shape of the sealing rubber directly affects the contact area between the sealing material and the upper and lower flanges on the application of the pretightening force and thus affects the sealing efficiency. The structures of the upper and lower flanges also affect the tightness of the hydraulic cylinder sealing structure. The size of the gap in the groove of the seal ring affects the amount of deformation of the seal rubber, 
which affects the sealing ability of the device. Chen et al. obtained an O-type seal with a higher reliability than the contact pressure distribution between the rectangular seal and the O-ring [11]. On the basis of the finite-element method, Zhou et al. proposed that reducing the height of the lower flange close to that of the measuring cavity of the oil cavity can reduce the loss of oil pressure and thus improve the sealing efficiency [12]. Lan et al. proposed a method of using finite sample points to establish a proxy model to conduct intradomain structural analysis and dimensional optimisation [13]; Wang et al. used genetic algorithm to optimise the size of the structure to improve the performance of the whole structure [14].

This study mainly used Ansys Workbench to perform finite-element analysis on the seals of hydraulic cylinders, compare the seal ring section shape, flange groove width, and groove depth, and optimise the groove structure through a surrogate model in MATLAB for obtaining a set of optimal structural parameters for the sealing rubber and flange and for achieving the best sealing effect for the hydraulic cylinder sealing structure.

\section{Model Description}

2.1. Geometric Model. The seal of a hydraulic cylinder has a typical symmetric structure. Considering the symmetry of the structures of the seal ring and hydraulic cylinder, this study simplified the three-dimensional plane strain problem for a hydraulic cylinder into a two-dimensional problem for simulation calculation. The two-dimensional axial seal models of the four seals considered in this study, which are based on the Chinese standard GB/T-3452.3-2005 [15], are displayed in Figure 1.

Figures 1(a) and 1(b) display single O-ring seal structures, and Figures 1(c) and 1(d) represent double O-ring seal structures $\left(A=\pi r^{2}\right)$. Figure 1(a) displays a schematic of a two-dimensional model of a seal groove with a gap, and Figure 1(b) displays a schematic of a two-dimensional model of a seal groove without a gap. Figure 1(c) depicts a schematic of a seal model with a gap, and Figure 1(d) depicts a schematic of a gapless seal model. In nonlinear finite-element simulation calculations, the following assumptions must be made to reduce the number of calculations and the calculation time:

(a) The rubber material is an isotropic and uniform material.

(b) The creep properties of rubber materials are uniform during elongation and compression, and the volume change is negligible when creep occurs.

(c) Compared with hydraulic cylinders, the quality of O-type rubber seals is negligible. The overall model of the hydraulic cylinder can be simplified into two dimensions.

2.2. Selection of Material Parameters. Because the sealing ring is usually made of rubber material and has a high degree of nonlinearity in the finite-element simulation, this study adopted the Mooney-Rivlin model with two parameters to represent the constitutive relationship of the material with large deformation [16]. The strain energy and compression constant in the aforementioned model can be expressed as follows:

$$
\begin{aligned}
& W=C_{1}\left(I_{1}-3\right)+C_{2}\left(I_{2}-3\right), \\
& D=\frac{(1-2 v)}{\left(C_{1}+C_{2}\right)},
\end{aligned}
$$

where $W$ is the strain energy; $D$ is the compression constant; $C_{1}$ and $C_{2}$ are the mechanical property constants of the nonlinear material; $I_{1}$ and $I_{2}$ are the first and second strain tensor invariants, respectively; and $v$ is Poisson's ratio of the material [17]. Theoretically, the mechanical property constant should be determined through tensile and compression experiments [18]; however, in practice, the constant is determined from the hardness value $(\mathrm{Hs})$ of the material $[19,20]$ :

$$
6 C_{1}\left(1+\frac{C_{1}}{C_{2}}\right)=\frac{15.75+2.15 H S}{100-H S} .
$$

The aforementioned formula indicates that the mechanical property constant of a material can be obtained from the $\mathrm{Hs}$ value and $\left(C_{1} / C_{2}\right)$. According to the literature [21], the recommended value of $\left(C_{1} / C_{2}\right)$ is generally 0.25 .

Considering the oil and water resistance, NBR is selected for analysing many rubber materials. The hardness of the seal under high pressure in this study was 85 . According to the aforementioned formula, $C_{1}=1.84 \mathrm{MPa}, C_{2}=0.47 \mathrm{MPa}$, $v=0.499$, and $D=0.00087 \mathrm{MPa}^{-1}$. The upper and lower flanges are made of standard structural steel, whose elastic modulus is $200 \mathrm{GPa}$ and Poisson's ratio is 0.3 .

Network segmentation is a critical step when a model is used for numerical simulation analysis. They must be as regular as possible in addition to the number of units that need to be controlled. Therefore, element independence analysis is usually required before determining the element size. As displayed in Figure 2, the maximum value of the contact pressure was stabilised at $4.38 \mathrm{MPa}$. Finally, the grid sizes of the upper and lower flanges and the sealing rubber were controlled to be less than $0.07 \mathrm{~mm}$. The grid of the hydraulic cylinder sealing system was divided into a combination of tetrahedrons and hexahedrons. The number of grids was 14,932, and the number of nodes was controlled at approximately 45,000 .

2.3. Contact Settings. Due to the large friction forces between the sealing rubber and the upper and lower flanges, the contact method was set to frictional contact, and the friction coefficient was $2 \times 10^{-2}$. The contact algorithm uses the augmented Lagrange multiplier method to adapt to largedeformation contact problems. Moreover, the finite-element control method uses the integration point detection method (Gauss Point) to analyse the friction contact problem [22, 23].

2.4. Boundary Condition Settings. In the finite-element analysis of the seal in this study, a $5.3 \mathrm{~mm}$-diameter nitrile rubber was used as the seal ring. The width $B$ of the seal groove with a gap was $6.3 \mathrm{~mm}$, and the width $\mathrm{B}$ of the seal 


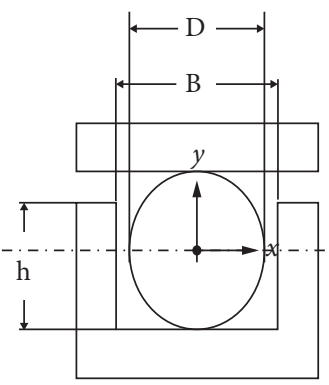

(a)

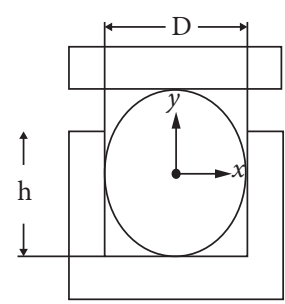

(b)

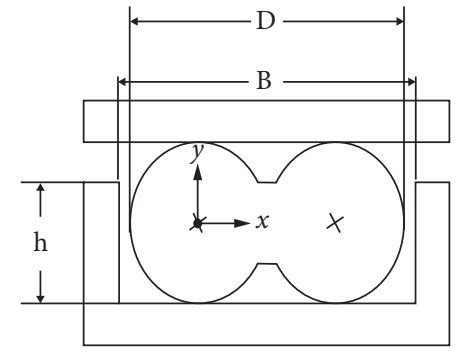

(c)

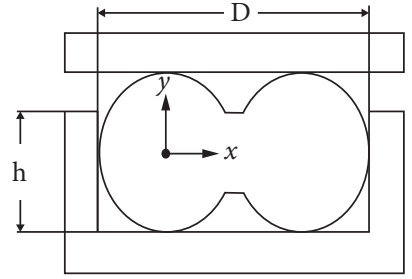

(d)

Figure 1: Two-dimensional models of the four seals considered in this study.

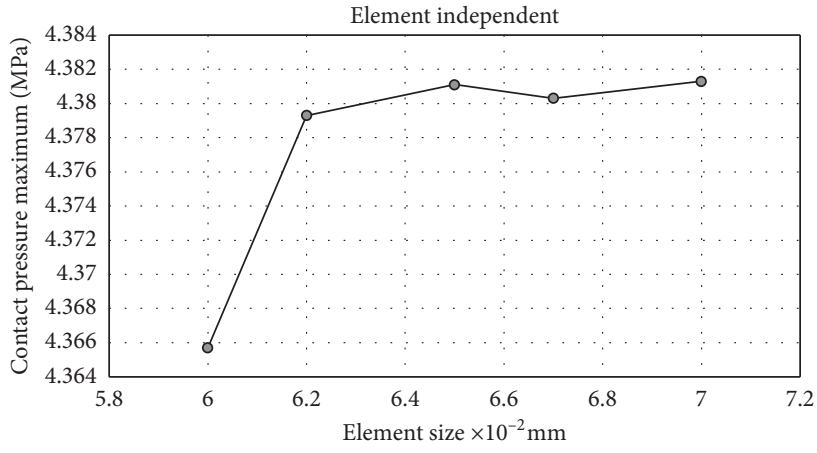

Figure 2: Element independence verification.

groove without a gap was $5.3 \mathrm{~mm}$. The seal groove depth of the single groove was $4.13 \mathrm{~mm}$. The first force causes large deformation, and second force constrains all the displacements of the lower flange. Three load steps were set. The first load step was the initial situation without any force; the second load step involved the application of a pretightening load, and the compression was $20 \%$ of the seal ring (i.e., $1.06 \mathrm{~mm}$ ); and the third load step involved setting the internal pressure of the hydraulic cylinder to $2 \mathrm{MPa}$. In the simulation, the left side was assumed as the inside of the hydraulic cylinder and the right side was assumed as the external side. Therefore, a pressure load of $2 \mathrm{MPa}$ was applied to the left side of the seal.

2.5. Simulation Results and Analysis. A comparison of the results obtained for a single-groove seal with and without gaps is displayed in Figure 3.

Figures 3(a) and 3(b) depict the equivalent stress cloud diagram of a single-groove seal with a gap and the cloud diagram of the contact pressure between this seal and the upper flange, respectively. Figures 3(c) and 3(d) depict the equivalent stress cloud diagram of the single-groove gapless seal and the cloud diagram of the contact pressure between this seal and the upper flange, respectively. Figures 3(e) and 3(f) illustrate the equivalent stress cloud diagram of a double-groove seal with a gap and the cloud diagram of the contact pressure between this seal and the upper flange, respectively. Figures 3(g) and 3(h) display the equivalent stress cloud diagram of the doublegroove gapless seal and the cloud diagram of the contact pressure between this seal and the upper flange, respectively.
The mean average pressure and maximum contact pressure results are presented in Table 1 . The mean equivalent stress was 2.66 MPa for the single-groove seals with and without a gap. Moreover, the difference in the maximum contact pressures of the aforementioned two seals was only $0.001 \mathrm{MPa}$. For the double-groove seal, the equivalent stresses with and without a gap were 2.65 and $2.64 \mathrm{MPa}$, respectively. Therefore, in the case of large compression, the presence or absence of gaps has almost no effect on the equivalent stress on the seal ring.

The contact pressure is the major factor affecting the sealing effect. When the contact pressures are greater than the pressures in the hydraulic cylinder, the sealing is successful and the sealing effect is proportional to the contact pressures. A comparison of Figures 3(b), 3(d), 3(f), and 3(h) indicates that the maximum contact pressures between the single-groove seal with a gap and the upper flange and between the double-groove seal with a gap and the upper flange were 4.3808 and $4.3876 \mathrm{MPa}$, respectively. The maximum contact pressures between the gapless singlegroove seal and the upper flange and between the gapless double-seal groove and the upper flange were 4.3813 and $4.3888 \mathrm{MPa}$, respectively. For single-groove seals, the maximum contact pressure without a gap was $0.011 \%$ higher than that with a gap. For double-groove seals, the maximum contact pressure without a gap was $0.17 \%$ higher than that with a gap. The maximum contact pressure was greater without a gap than with a gap because the horizontal deformation of gapless seal grooves was constrained. The aforementioned data indicate that the number of seal ring layers considerably influences the sealing effect of the hydraulic cylinder for the adopted two sets of variables. Therefore, structure optimisation was performed for the seal groove and seal ring of the double-layer seal without a gap.

\section{Structural Optimisation Design Based on a Surrogate Model}

Structural optimisation design involves determining a set of optimal solutions from design variables under the proposed constraints. The general optimisation solution process involves mathematically modelling the optimisation problem, proposing constraints according to the mathematical model, and selecting an appropriate method to optimise the established model for a certain goal. In general, the following parameters are considered in the process of optimal design [24]: 
Unit (MPa)

\begin{tabular}{|l}
$4.2228 \mathrm{Max}$ \\
3.7553 \\
3.2878 \\
2.8203 \\
2.3528 \\
1.8854 \\
1.4179 \\
0.95039 \\
0.48291 \\
$0.01542 \mathrm{Min}$
\end{tabular}

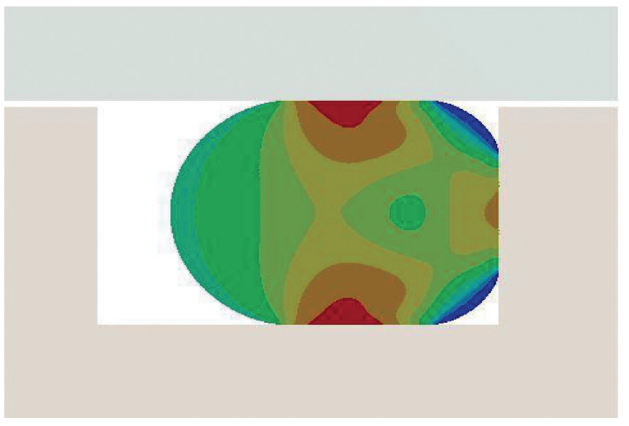

(a)
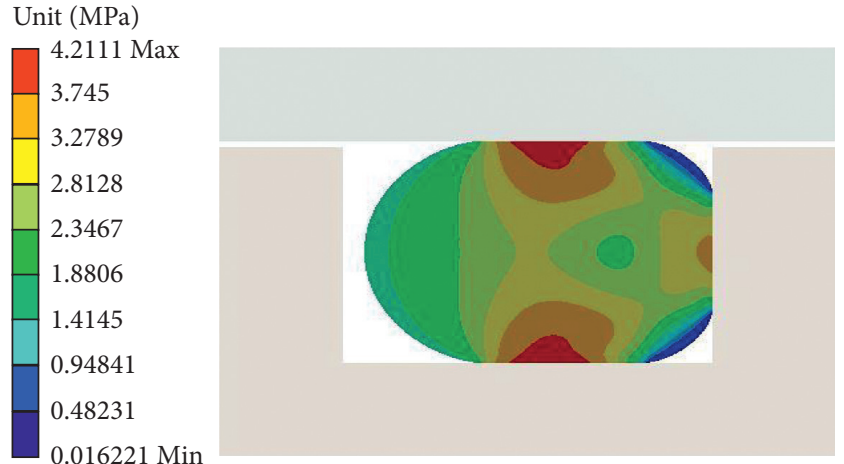

(c)

Unit (MPa)
\begin{tabular}{|l}
$4.626 \mathrm{Max}$ \\
- \\
-1.1134 \\
3.6008 \\
3.0881 \\
2.5755 \\
-2.0629 \\
1.5503 \\
1.0376 \\
0.52499 \\
$0.012359 \mathrm{Min}$
\end{tabular}

\begin{tabular}{|}
$4.6918 \mathrm{Max}$ \\
4.1717 \\
3.6516 \\
3.1316 \\
2.6115 \\
2.0915 \\
1.5714 \\
1.0513 \\
0.53126 \\
$0.011191 \mathrm{Min}$
\end{tabular}

(e)

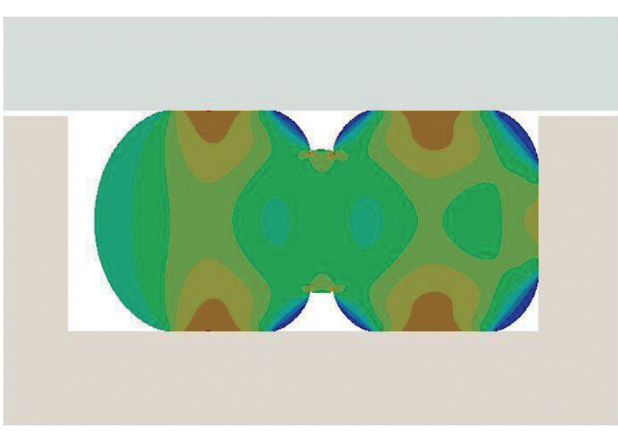

Unit (MPa)
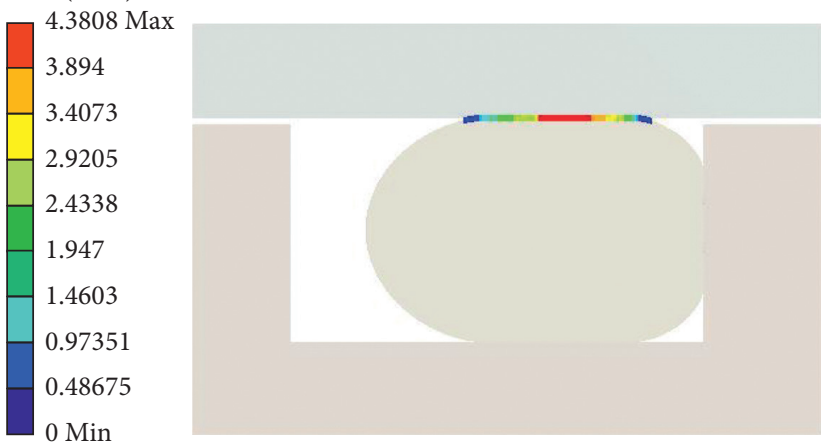

(b)
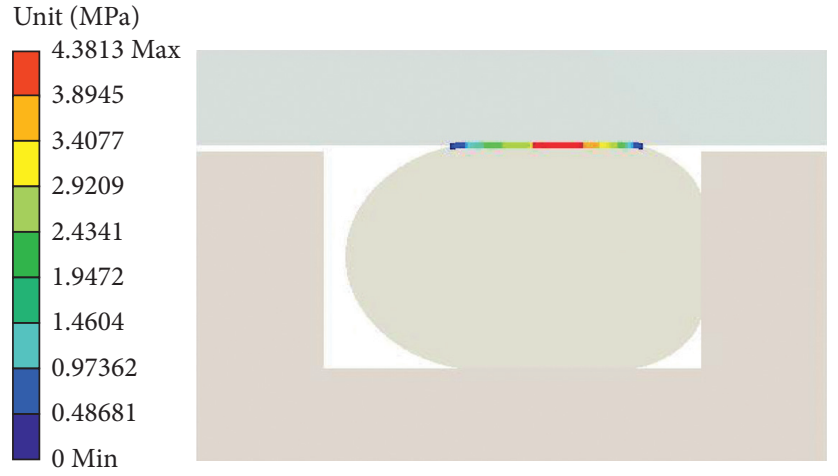

(d)

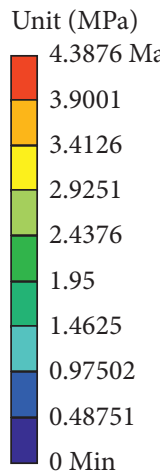

(g)
Unit (MPa)
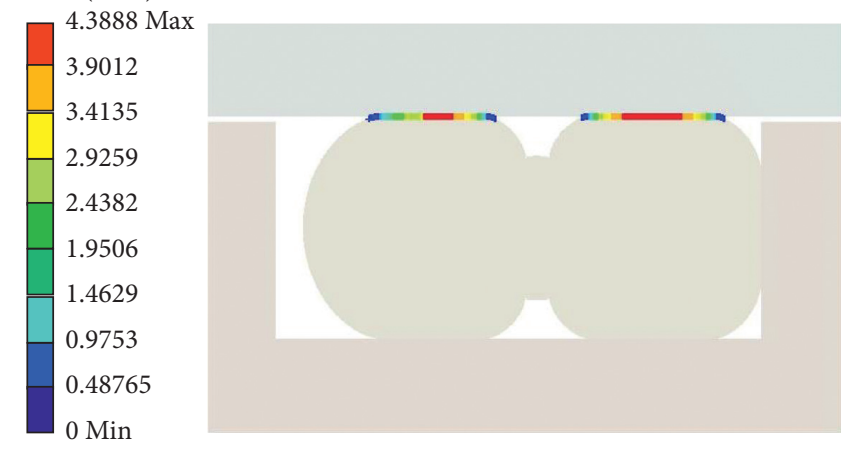

(h)

Figure 3: Equivalent stress and contact pressure cloud diagrams of the single-groove seal. 
TABLE 1: Mean average pressure and maximum contact pressure for different sealing methods.

\begin{tabular}{lcccc}
\hline & $\begin{array}{c}\text { Single seal with gap } \\
(\mathrm{MPa})\end{array}$ & $\begin{array}{c}\text { Single seal with gapless } \\
(\mathrm{MPa})\end{array}$ & $\begin{array}{c}\text { Double seal with gap } \\
(\mathrm{MPa})\end{array}$ & $\begin{array}{c}\text { Double seal with gapless } \\
(\mathrm{MPa})\end{array}$ \\
\hline $\begin{array}{l}\text { Mean equivalent stress } \\
\begin{array}{l}\text { Maximum contact } \\
\text { pressure }\end{array}\end{array}$ & 2.66 & 2.66 & 2.65 & 2.65 \\
\hline
\end{tabular}

(a) Design variables: design variables are parameters that have a major influence on the model (design variables are generally denoted by $x$ ).

(b) Objective function: the objective function is the optimisation objective (optimisation object), which is generally denoted as $f(x)$.

(c) Constraints: constraints refer to the ranges of design variables. The types of constraints in the optimisation process include stress and volume constraints.

3.1. Determination of Design Variables. According to the simulation results presented in Section 2.5, the double-layer seal without a gap exhibits a superior sealing effect to the other seal structures. Therefore, the double-layer seal structure was parameterised and modelled, as depicted in Figure 1(d). Two parameters, namely, the diameter of the seal ring $(D)$ and the depth of the seal groove $(h)$, were calculated due to their high influence on the sealing effect in parametric modelling. In addition, the chamfer of the upper and lower flanges affects the equivalent stress on the seal ring. However, in currently used structures, the seal ring does not contact the chamfer of the flange under a compression of $20 \%$ and working pressure of $2 \mathrm{MPa}$. Therefore, the chamfer was not considered as a design variable in the study [25].

3.2. Determination of the Objective Function. In this study, the maximum value of the contact pressure between the seal ring and the upper flange was used to evaluate the sealing effect [26].

3.3. Determination of Constraints. The current diameter of the seal ring was $5.3 \mathrm{~mm}$, and the diameter of the seal ring (D) was set to $4-7 \mathrm{~mm}$ according to the sealing conditions considered in this study. The depth of the seal groove $(h)$ must not be less than the radius of the seal ring. Moreover, sufficient displacement must be caused by the upper flange due to the pretightening force [27]. Therefore, the mathematical model of the seal structure is expressed as follows:

$$
\begin{array}{ll}
\text { Find } & X=[D, h]^{T}, \\
\max & f(x)=f(D, h),
\end{array}
$$$$
\text { Subject to } 4 \mathrm{~mm} \leq D \leq 7 \mathrm{~mm} \text {, }
$$$$
\frac{D}{2} \leq h \leq D-1.2 \mathrm{~mm}
$$

3.4. Test Design. The Latin hypercube method [28, 29] was used to obtain the test points in the experiment. The points selected using this method can evenly cover the entire design space. Considerable space information can be accurately obtained with only a small number of test points. Because the upper and lower limits of the design variables in this study changed with a change in any parameter, a constrained Latin hypercube was used to select the test point [30].

The number of test points was selected according to the following principle: the number of test points must be 10 times higher than the number of variables. Two design variables were used in this study; therefore, 20 points were selected to build the surrogate model. The plane distribution of the design variables is displayed in Figure 4, in which the value ranges of the design variables are indicated by the coordinate axes.

3.5. Parametric Modelling. After the selection of the test points, the maximum contact pressure between the sealing ring and the upper flange was calculated for all the training and test points through finite-element simulation. Ansys Workbench was directly used for parametric modelling and finite-element calculation. The corresponding results are presented in Table 2.

3.6. Surrogate Model. The interpolating recmultiquadric radial basis function method [31-33] was used for data fitting after obtaining the design and response points. A total of $20 \%$ of the data points (five points) were used as test points. The response surface and residuals of the training points are displayed in Figure 5.

The residual error of the training points was $10^{-7}$ (Figure 5), which is considerably lower than $10^{-4}$; thus, the surrogate model had a high degree of fit for the training points. The root mean square error of the aforementioned model was 0.48 for the five test points. The difference between the maximum stress values obtained with the surrogate model and in the experiment $(7.3 \mathrm{MPa})$ was less than $10 \%$, which is within the acceptable error range.

3.7. Optimisation of the End Plate Structure by Using the Genetic Algorithm. The genetic algorithm [34, 35] was used to optimise the design of the seal structure. The final iteration results are presented in Table 3. The maximum contact pressure was 7.28 $\mathrm{MPa}$ [36].

3.8. Reliability of the Sealing Structure. Let $X_{1}, X_{2}, \ldots, X_{n}$ be $n$ random variables that affect the structural function, the function of which can be expressed as the following equation: 


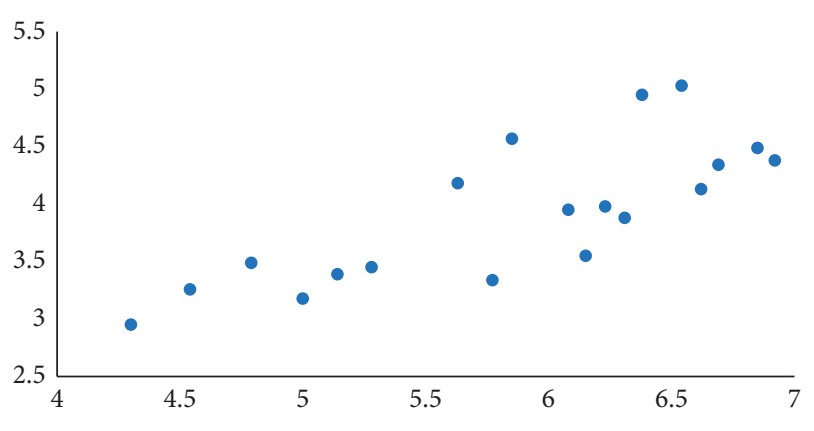

Figure 4: Plane distribution of design variables.

TABLE 2: Data points and response values.

\begin{tabular}{lcc}
\hline$D(\mathrm{~mm})$ & $h(\mathrm{~mm})$ & $P_{\max }(\mathrm{MPa})$ \\
\hline 5.14 & 3.39 & 6.528 \\
6.23 & 3.98 & 4.1881 \\
4.54 & 3.26 & 4.8721 \\
5.28 & 3.45 & 6.4509 \\
6.31 & 3.88 & 4.1706 \\
6.85 & 4.49 & 4.027 \\
5.77 & 3.34 & 4.3518 \\
5.85 & 4.57 & 4.302 \\
5.63 & 4.18 & 4.3994 \\
6.92 & 4.38 & 4.015 \\
5.00 & 3.18 & 6.3237 \\
6.62 & 4.13 & 4.0882 \\
6.15 & 3.55 & 4.2387 \\
6.69 & 4.34 & 4.0658 \\
6.08 & 3.95 & 4.234 \\
7.00 & 5.79 & 3.992574 \\
4.30 & 2.95 & 5.0262 \\
6.54 & 5.03 & 4.1055 \\
6.38 & 4.95 & 4.1484 \\
4.79 & 3.49 & 6.5858 \\
\hline
\end{tabular}

$$
Z=g\left(X_{1}, X_{2}, \ldots, X_{n}\right)
$$

where $X_{1}, X_{2}, \ldots, X_{n}$ could be the structural dimension parameter, the physical property of the material, or the loading on the structure. When $Z>0$, the structure could perform the objective functions and work in a reliable state; when $Z<0$, the structure could fail to perform the objective function and become unstable; when $Z=0$, the structure is in the limit state or critical state. In this paper, $p_{f}$ denoted the probability of failure, i.e., the probability of the structural function $Z<0$. We used $f(z)$ to represent the probability density function. Therefore, the probability of $Z<0$ is

$$
p_{f}=\int_{-\infty}^{0} f(z) \mathrm{d} z \text {. }
$$

However, the structure function should be standardized in the actual calculations:
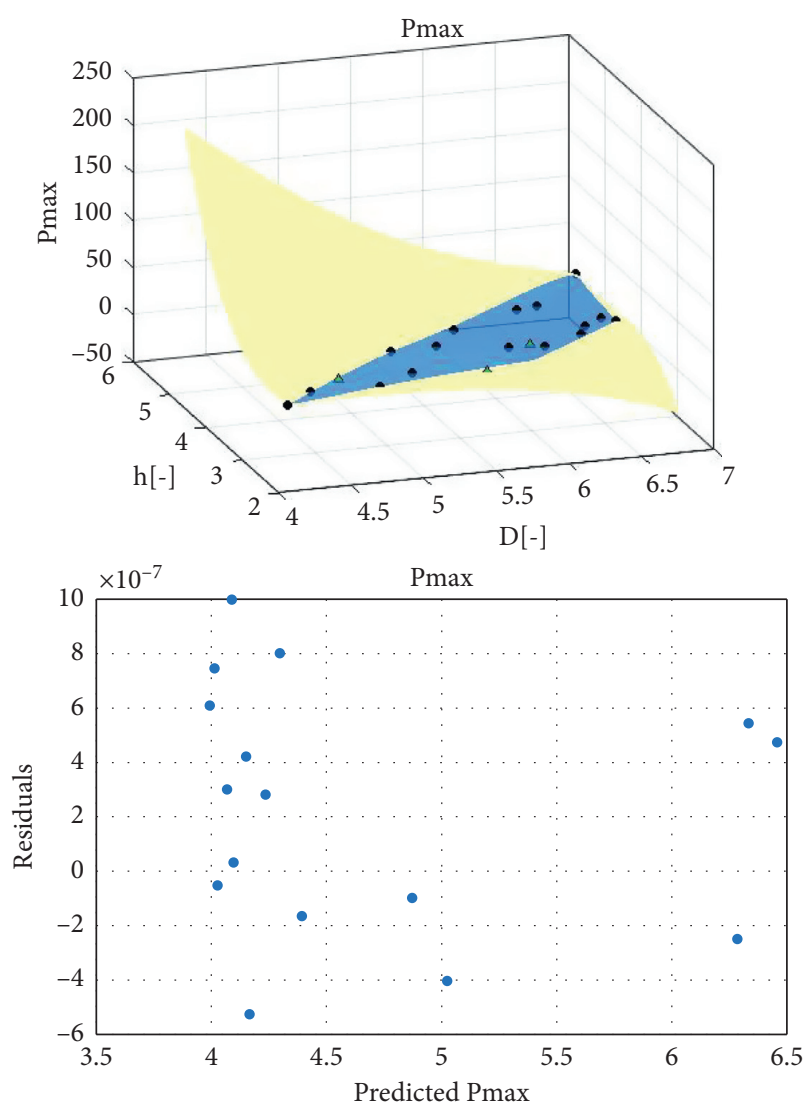

FIGURE 5: Response surface and residuals of the training points.

TABLE 3: Optimal design variables and optimal solutions.

\begin{tabular}{lcc}
\hline$D(\mathrm{~mm})$ & $h(\mathrm{~mm})$ & $P_{\max }(\mathrm{MPa})$ \\
\hline 4.23 & 2.8 & 7.2778 \\
& $Z^{\prime}=\frac{C-\mu_{z}}{\sigma_{z}}$.
\end{tabular}

The probability of failure after standardization can be expressed as

$$
p_{f}=\int_{-\infty}^{\left(-\mu_{z} / \sigma_{z}\right)} f\left(z^{\prime}\right) \mathrm{d} z^{\prime}
$$

The reliability of the structure can be expressed as

$$
P_{r}=1-P_{f}
$$

In this paper, the Gram-Charlier series was used to decompose and fit the distribution of the function, which could be expressed as

$$
f(X)=\Phi \prime(X)=\sum_{n=3}^{\infty} \frac{(-1)^{n} C_{n}}{\sqrt{n !}} \Phi^{(n+1)}(X),
$$


TABLE 4: The mean values and the mean square error in case of the maximum contact pressure after storage and aging of the sealed structure.

\begin{tabular}{lccccc}
\hline Time of usage & Shortly after sealing & 10 years & 20 years & 25 years & 30 years \\
\hline Mean of response $(\mathrm{MPa})$ & 4.59 & 4.57 & 4.38 & 3.72 & 3.64 \\
Mean square error of response $(\mathrm{MPa})$ & 0.94 & 0.88 & 0.84 & 0.72 & 0.72 \\
\hline
\end{tabular}

TABLE 5: Reliability of 5 sealed structure joints.

\begin{tabular}{|c|c|c|c|c|c|}
\hline Serial number & Shortly after sealing & 10 years & 20 years & 25 years & 30 years \\
\hline 1 & 0.976 & 0.921 & 0.851 & 0.827 & 0.796 \\
\hline 2 & 0.983 & 0.926 & 0.846 & 0.821 & 0.793 \\
\hline 3 & 0.984 & 0.927 & 0.859 & 0.835 & 0.794 \\
\hline 4 & 0.987 & 0.931 & 0.861 & 0.845 & 0.807 \\
\hline 5 & 0.994 & 0.938 & 0.881 & 0.848 & 0.813 \\
\hline
\end{tabular}

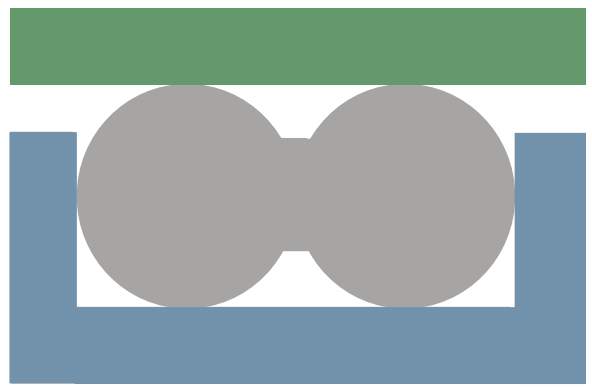

(a)
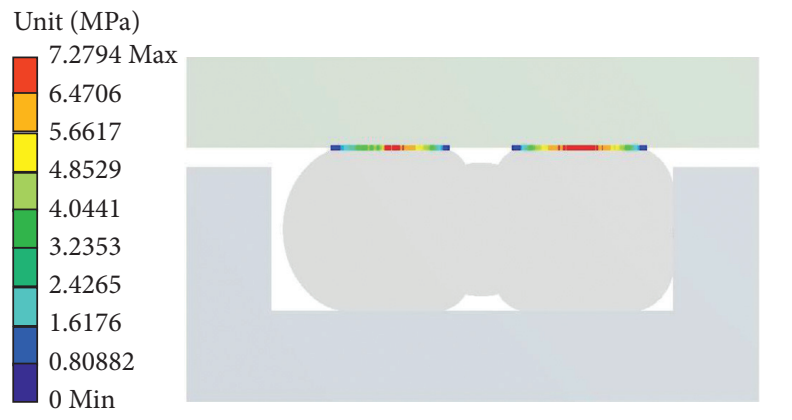

(c)

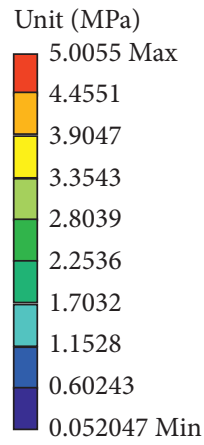

Unit (MPa)
\begin{tabular}{|l}
$2.654 \mathrm{Max}$ \\
2.3624 \\
2.0707 \\
1.7791 \\
1.4874 \\
1.1958 \\
0.90415 \\
0.6125 \\
0.32086 \\
$0.029211 \mathrm{Min}$
\end{tabular}

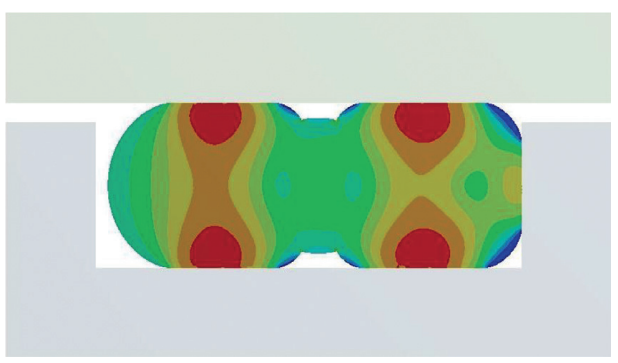

(d)

FIGURE 6: Model and simulation results.

where $\Phi(X)=(1 / \sqrt{2 \pi}) \int_{0}^{x} e^{-\left(t^{2} / 2\right)} \mathrm{d} t$ and $C_{n}$ is the moment coefficient of each order. In this paper, in order to improve the calculation accuracy, the order of six was selected for calculation, which revealed the following equations:

$$
\begin{gathered}
C_{0}=1, \\
C_{1}=C_{2}=1, \\
C_{3}=\frac{1}{\sqrt{3 !}} \frac{\mu_{3}}{\sigma^{3}}, \\
C_{4}=\frac{1}{\sqrt{4 !}}\left(\frac{\mu_{4}}{\sigma^{4}}-3\right), \\
C_{5}=\frac{1}{\sqrt{5 !}}\left(\frac{\mu_{5}}{\sigma^{5}}-10 \frac{\mu_{3}}{\sigma^{3}}\right),
\end{gathered}
$$

$$
C_{6}=\frac{1}{\sqrt{5 !}}\left(\frac{\mu_{6}}{\sigma^{6}}-15 \frac{\mu_{4}}{\sigma^{4}}+30\right)
$$

where $\mu_{k}$ is the $k$ th central moment of $Z$, and $\sigma^{k}$ is the $k$ th power of the mean square error of $Z$ '.

According to the finite-element analysis results above, the mean values and the mean square error in case of the maximum contact pressure after storage and aging of the sealed structure can be calculated. The results are shown in Table 4.

According to the finite-element simulation and the surrogate model, the limit state equation of the sealing structure was established, and the corresponding moments of the structure under the maximum contact pressure in each aging period can be obtained, and the reliability of the sealing structure can be calculated according to the above equations. In this paper, five nodes in the middle of the 
TABLE 6: Comparison of the optimised and unoptimised results.

\begin{tabular}{lccc}
\hline & Unoptimized seal structure $(\mathrm{MPa})$ & Optimized sealing structure $(\mathrm{MPa})$ & Lift percentage $(\%)$ \\
\hline Mean equivalent stress & 2.65 & 5.01 & 89.06 \\
Maximum contact pressure & 4.39 & 7.28 & 65.83 \\
Maximum shear stress & 2.67 & 2.65 & -0.75 \\
\hline
\end{tabular}

O-ring were selected for calculation. The results of reliability for the sealing structure joints are shown in Table 5 .

The average value of the five nodes was used to represent the reliability of the sealing structure; it can be seen that the reliability of the sealing structure after 10 years, 20 years, 25 years, and 30 years was $0.985,0.929,0.859$, and 0.801 , respectively. A failure rate less than $10 \%$ is acceptable for ordinary hydraulic cylinder sealing structures. Therefore, the sealing structure could still be effective after 10 years.

\section{Comparison of the Results Obtained before and after Optimisation}

The parameters obtained after design variable optimisation were substituted into the model, and simulation was performed using Ansys Workbench. The maximum contact pressure was obtained as $7.2794 \mathrm{MPa}$. The error between the aforementioned value and the corresponding result obtained using the surrogate model was $0.027 \%$. The model and simulation results are presented in Figure 6.

Figure 6(a) displays a schematic of the optimised seal model, and Figure 6(b) displays the equivalent stress cloud diagram. Figure 6(c) illustrates the cloud diagram of contact pressure between the seal ring and the upper flange, and Figure 6(d) displays the maximum shear stress cloud diagram. A comparison of the optimised and unoptimised structural design results are presented in Table 6.

According to the aforementioned simulation results, all the structures, the O-ring seal did not contact the chamfers of the seal grooves under a preload of $1.06 \mathrm{~mm}$ and working pressure of $2 \mathrm{MPa}$. The equivalent stress had a spindleshaped distribution and was larger at the contact area than at other areas. The average equivalent stress of the optimised structure was $89.06 \%$ higher than that of the unoptimised structure. Moreover, the maximum contact pressure for the optimised structure was $65.83 \%$ higher than that for the unoptimised structure. The maximum shear stress indicates the service life of the seal ring. The maximum shear stress of the optimised seal structure was $0.75 \%$ lower than that of the unoptimised structure; thus, the shear stress was only marginally reduced after optimisation. The aforementioned results indicate that the optimised structure has a considerably stronger sealing effect and only marginally smaller service life than the original structure.

\section{Conclusions}

(a) The obtained qualitative finite-element simulation results indicate that, during the operation of hydraulic cylinders, the peak values of the equivalent stress and contact pressure are always located at the contact between the seal ring and the upper flange in the prestressed state. Thus, the sealing effect of a hydraulic cylinder seal can be assessed according to the maximum contact pressure at the contact between the seal and the upper flange.

(b) The finite-element analysis results obtained for the four sealing structures considered in this study indicated that the sealing effect of the gapless double $\mathrm{O}$-ring was stronger than those of the double O-ring with a gap, the gapless single $\mathrm{O}$-ring, and the single O-ring with a gap.

(c) According to the surrogate model and finite-element simulation calculations, the contact pressure of the optimised seal structure is $65.83 \%$ higher than that of the original gapless double $\mathrm{O}$-ring. The combination of the developed surrogate model and finite-element method can provide ideas for the optimal design of seal structures without sacrificing the service life.

\section{Data Availability}

The datasets supporting the conclusions of this study are included within the article.

\section{Conflicts of Interest}

The authors declare that there are no conflicts of interest regarding the publication of this paper.

\section{Acknowledgments}

This project was supported by National Natural Science Foundation of China (Grant no. 51975092)

\section{References}

[1] Y.-F. Gu, Y. Zhao, R.-Q. Lv, and Y. Yang, "A practical FBG sensor based on a thin-walled cylinder for hydraulic pressure measurement," IEEE Photonics Technology Letters, vol. 28, no. 22, pp. 2569-2572, 2016.

[2] L. Solazzi and A. Buffoli, "Telescopic hydraulic cylinder made of composite material," Applied Composite Materials, vol. 26, no. 4, pp. 1189-1206, 2019.

[3] C. Zhan, "Research on low-friction and high-response hydraulic cylinder with variable clearance," Journal of Mechanical Engineering, vol. 51, no. 24, p. 161, 2015.

[4] G. H. Han and Y. L. Fu, "A study on the accelerated life test for hydraulic cylinders," Advanced Materials Research, vol. 199200, pp. 630-637, 2011.

[5] R. Lin, Y. Guo, S. Wei et al., "Critical load and optimum design for hydraulic cylinders," China Mechanical Engineering, vol. 22, no. 4, pp. 389-393, 2011.

[6] C. H. Chen, C. T. Zhong, J. B. Wan et al., "Simulation and design for the hydraulic synchronization control system of clamping cylinders of tensioner on deep water cable-laying 
vessel," Ocean Engineering, vol. 30, no. 4, pp. 137-143, 2012.

[7] M. Kamezaki, H. Iwata, and S. Shigeki, "A practical approach to detecting external force applied to hydraulic cylinder for construction manipulator," in SICE Annual Conference, IEEE, Taipei, Taiwan, August 2010.

[8] X. Sheng, G. Si, and X. Li, "Reconfigurable hydraulic-mechanical combined drive devices based on multi-functional piston-rod built-in hydraulic cylinder," in Proceedings of 2010 International Conference on Digital Manufacturing \& Automation, IEEE, Changsha, China, December 2010.

[9] E. Kassfeldt, "Analysis and design of hydraulic cylinder seals," 2016.

[10] F. Zhang, "The innovative design of reciprocating seal for hydraulic cylinder based on triz evolution theory," in Proceedings of International Conference on Programming Languages for Manufacturing, pp. 440-449, Tianjin, China, 2006.

[11] R. Chen, "The sealing problem of solid engine," Strength and Environment, vol. 4, pp. 1-5, 1995.

[12] Y. Zhou, B. Sun, S. Wang et al., "Finite element analysis of hydraulic cylinder sealing performance and friction force," Coal Mining Machinery, vol. 39, no. 03, pp. 50-53, 2018.

[13] H. Lan, L. Yang, F. Zhang, C. Zong, S. Wi, and X. Song, "Analysis and optimization of high temperature proton exchange membrane (HT-PEM) fuel cell based on surrogate model," International Journal of Hydrogen Energy, vol. 45, no. 22, pp. 12501-12513, 2020.

[14] H.-R Wang, S Wu, L.-L Yang et al., “Topology optimization of high temperature proton exchange membrane fuel cell reactor end plate," Journal of Dalian University of Technology, vol. 60, no. 02, pp. 142-148, 2020.

[15] X. Chen, Finite Element Analysis and Optimization Design of High-Pressure Vessel Sealing Structure, Lanzhou University, Lanzhou, China, 2015.

[16] M. Yamamoto, S. Minewaki, H. Yoneda et al., "Nonlinear behavior of high-damping rubber bearings under horizontal bidirectional loading: full-scale tests and analytical modeling," Earthquake Engineering \& Structural Dynamics, vol. 41, no. 13, pp. 1845-1860, 2012.

[17] Y. Ma, F. Scarpa, D. Zhang et al., "A nonlinear auxetic structural vibration damper with metal rubber particles," Smart Materials and Structures, vol. 22, no. 8, Article ID 084012, 2013.

[18] L. G. Maqueda, A. N. A. Mohamed, and A. A. Shabana, "Use of general nonlinear material models in beam problems: application to belts and rubber chains," Journal of Computational and Nonlinear Dynamics, vol. 5, no. 2, 2010.

[19] H. Wang, J. A. Rongong, G. R. Tomlinson et al., "Nonlinear static and dynamic properties of metal rubber dampers," Energy, vol. 10, no. 1, 2010.

[20] N. Koprowski-Theiß, M. Johlitz, and S. Diebels, "Modelling of a cellular rubber with nonlinear viscosity functions," Experimental Mechanics, vol. 51, no. 5, pp. 749-765, 2011.

[21] Z. Liang and B. Xiao, "A method for determining the material coefficient of the rubber," Mooney-Rivlin Model, vol. 46, no. 527, pp. 38-40, 2008.

[22] R. A. Hawileh, A. Rahman, and H. Tabatabai, "Nonlinear finite element analysis and modeling of a precast hybrid beam-column connection subjected to cyclic loads," Applied Mathematical Modelling, vol. 34, no. 9, pp. 2562-2583, 2010.

[23] S. R. Wu and L. Gu, Introduction to the Explicit Finite Element Method for Nonlinear Transient dynamics, John Wiley \& Sons, Hoboken, NJ, USA, 2012.
[24] M. C. H. Wu, D. Kamensky, C. Wang et al., "Optimizing fluid-structure interaction systems with immersogeometric analysis and surrogate modeling: application to a hydraulic arresting gear," Computer Methods in Applied Mechanics and Engineering, vol. 316, pp. 668-693, 2017.

[25] F. Sanchez, M. Budinger, and I. Hazyuk, "Dimensional analysis and surrogate models for the thermal modeling of Multiphysics systems," Applied Thermal Engineering, vol. 110, pp. 758-771, 2017.

[26] R. Mallipeddi and M. Lee, "An evolving surrogate modelbased differential evolution algorithm," Applied Soft Computing, vol. 34, pp. 770-787, 2015.

[27] W. Yamazaki, M. Rumpfkeil, and D. Mavriplis, "Design optimization utilizing gradient/hessian enhanced surrogate model," in Proceedings of 28th AIAA Applied Aerodynamics Conference, p. 4363, January 2010.

[28] H. Dette and A. Pepelyshev, "Generalized Latin hypercube design for computer experiments," Technometrics, vol. 52, no. 4, pp. 421-429, 2010.

[29] F. Zhang, L. Cheng, Y. Gao et al., "Fault tree analysis of a hydraulic system based on the interval model using latin hypercube sampling," Journal of Intelligent \& Fuzzy Systems, pp. 1-11, 2019.

[30] P. Z. G. Qian, "Sliced Latin hypercube designs," Journal of the American Statistical Association, vol. 107, no. 497, pp. 393399, 2012

[31] B. Sarler, N. Massarotti, P. Nithiarasu et al., "Local radial basis function collocation method for linear thermoelasticity in two dimensions," International Journal of Numerical Methods for Heat \& Fluid Flow, vol. 25, no. 6, pp. 1488-1510, 2015.

[32] O. Estruch, O. Lehmkuhl, R. Borrell et al., "A parallel radial basis function interpolation method for unstructured dynamic meshes," Computers \& Fluids, vol. 80, pp. 44-54, 2013.

[33] X. Yang, B. Xue, L. Jia et al., "Quantitative analysis of pit defects in an automobile engine cylinder cavity using the radial basis function neural network-genetic algorithm model," Structural Health Monitoring, vol. 16, no. 6, pp. 696-710, 2017

[34] B. Mhamdi, K. Grayaa, and T. Aguili, "Hybrid of genetic algorithm with particle swarm optimization to shape reconstruction of perfect conducting cylinders," AEU-international Journal of Electronics and Communications, vol. 65, no. 12, pp. 1032-1039, 2011.

[35] B. Mhamdi, K. Grayaa, and T. Aguili, "Microwave imaging of dielectric cylinders from experimental scattering data based on the genetic algorithms, neural networks and a hybrid micro genetic algorithm with conjugate gradient," AEU-international Journal of Electronics and Communications, vol. 65, no. 2, pp. 140-147, 2011.

[36] G. Raju, B. Panitapu, and S. Naidu, "Optimal design of an IC engine cylinder fin arrays using a binary coded genetic algorithms," International Journal of Modern Engineering Research, vol. 2, no. 6, pp. 4516-4520, 2012. 